\title{
Anti-metastasis efficacy and safety of non-anticoagulant heparin derivative versus low molecular weight heparin in surgical pancreatic cancer models
}

\author{
REEM ALYAHYA ${ }^{1,2^{*}}$, THANGIRALA SUDHA ${ }^{1 *}$, MICHAEL RACZ $^{3}$, STEVEN C. STAIN $^{4}$ and SHAKER A. MOUSA ${ }^{1}$ \\ ${ }^{1}$ The Pharmaceutical Research Institute, Albany College of Pharmacy and Health Sciences, Rensselaer, \\ NY, USA; ${ }^{2}$ Department of Surgery, King Saud University, Riyadh, Saudi Arabia; ${ }^{3}$ Department of Basic and \\ Social Sciences, Albany College of Pharmacy and Health Sciences, Albany, NY, USA; \\ ${ }^{4}$ Department of Surgery, Albany Medical College, Albany, NY, USA
}

Received October 9, 2014; Accepted November 27, 2014

DOI: $10.3892 /$ ijo.2014.2803

\begin{abstract}
Heparin and its derivatives are known to attenuate cancer metastasis in preclinical models, but have not been used clinically due to adverse bleeding effects. This study compared the efficacy of S-NACH (a sulfated non-anticoagulant heparin) versus tinzaparin (a low molecular weight heparin) in inhibiting metastasis of a growing primary tumor and following surgical excision of primary tumor in a pancreatic cancer mouse model. The efficacy of S-NACH versus tinzaparin on metastasis of the primary tumor was evaluated in each experiment using IVIS imaging. Athymic female mice were treated with S-NACH or tinzaparin, and $30 \mathrm{~min}$ later luciferase-transfected pancreatic cancer cells (Mpanc96) were implanted into the spleen; treatment was continued daily until termination. Next we studied the effect of S-NACH versus tinzaparin on metastasis after surgical excision of the primary tumor after 3 weeks of daily treatment with S-NACH or tinzaparin. S-NACH reduced surgically induced metastasis $(\mathrm{p}<0.01)$ and tumor recurrence $(\mathrm{p}<0.05)$ relative to control. Histopathological studies demonstrated significant increase in tumor necrosis mediated by S-NACH and to lesser extent by tinzaparin as compared to control group. Furthermore, either S-NACH or tinzaparin upregulated the expression of the junctional adhesion molecule E-cadherin in pancreatic cancer cells where its low expression enhances cancer cell migration and invasion. In terms of bleeding time (BT), S-NACH did not affect BT as compared to tinzaparin, which doubled BT.
\end{abstract}

Correspondence to: Dr Shaker A. Mousa, The Pharmaceutical Research Institute, Albany College of Pharmacy and Health Sciences, 1 Discovery Drive, Rensselaer, NY 12144, USA

E-mail: shaker.mousa@acphs.edu

${ }^{*}$ Contributed equally

Key words: cancer metastasis, LMWH, sulfated non-anticoagulant heparin, pancreatic cancer, platelet, selectins, E-cadherin
These data suggest that S-NACH is an effective and safe antimetastatic agent and warrants further clinical evaluation.

\section{Introduction}

Pancreatic cancer is the fourth-leading cause of cancer-related deaths in both males and females in the United States (1), and it has been postulated that the primary cause of death in cancer patients is due to the consequences of metastasis (2). The overall 5-year survival rate for over 100,000 pancreatic cancer patients diagnosed from $1985-1995$ was $23.4 \%$ for patients who had surgical treatment due to metastasis or cancer resurgence (3). Heparin is an efficient anti-metastatic agent; it inhibits metastasis by binding to P-selectin and blocking the adhesion between platelets and the disseminated tumor cells in the blood. The use of heparin as an anti-metastatic agent is limited due to the side effect of bleeding. In our experiments we aimed to develop and test the inhibitory effect of heparin derivatives that are devoid of systemic anticoagulation on blood-borne metastasis and on surgically induced metastasis.

$\mathrm{P}$-selectin is among the selectins in a family of cell adhesion molecules divided into groups E, L, and P found on the surface of endothelial cells, leukocytes, and platelets, respectively, and has been studied extensively. P-selectin was found to be most relevant in the process of tumor metastasis. The adhesions that form between tumor cells and platelets via P-selectin are required to create the metastatic micro-thrombi (4). Sialylated fucosylated glycans are the ligands for P-selectin, and tumors with high expression of these ligands typically have poor prognosis due to high rates of metastasis $(5,6)$.

Heparin was found to be an efficient ligand for P-selectin and blocks its binding with tumor cells, and therefore attenuates tumor metastasis in animal models $(7,8)$. By depriving the circulating tumor cells of their platelet shield, they become more fragile in the harsh environment of the circulatory system and are more readily cleared by the immune system. The anti-metastatic properties of heparin are a result of one or more of the following: inhibition of heparinase, blocking of P- and L-selectins (9), inhibition of tissue factor (10) and inhibition of angiogenesis (11). 
Low molecular weight heparin (LMWH) has been shown to decrease tumor metastasis in animal experiments and clinical trials (12), but the use of heparin and its LMWH derivatives as anti-metastatic agents is limited because of the risk of inducing adverse bleeding complications. A meta-analysis performed in 2007 showed an increase in bleeding in patients treated with LMWH as an anti-metastatic (12). To overcome this complication, we developed sulfated non-anticoagulant heparin (NACH) derivatives (13). Several experimental studies have shown that certain heparin derivatives devoid of systemic anticoagulant activities can reduce the incidence of experimental metastasis (14). Hence, $\mathrm{NACH}$ is expected to have the same anti-metastatic effect as LMWH because it carries all properties of heparin except the anticoagulant activity. In our laboratory we developed a novel sulfated form of $\mathrm{NACH}$, named S-NACH, and it was found to be effective and safe in a mouse model of tumor growth and tumor angiogenesis (15).

Tumor excision of a primary cancer with no metastasis has been the cornerstone treatment for most cancers, but scientific evidence has revealed that tumor manipulation during resection can increase the risk of metastasis (16). Clinical research has shown that heparin is effective in reducing metastasis in surgical patients. In 1995 a study was done to evaluate the use of heparin to reduce surgically induced venous thromboembolism. Re-analysis of the survival data comparing patients who received heparin to those who did not showed that the threeyear mortality from disseminated malignancy was reduced in half (9.2 vs. 21.4\%) (17). In 2000, von Tempelhoff et al studied the effect of the LMWH certoparin in a randomized, double blind study of ovarian cancer patients with follow-up for 2 years after surgery (18). The death rate with administration of certoparin vs. unfractionated heparin was 21.4 vs. $37.5 \%$ deaths, respectively, at 2 years.

In our current investigation, we aimed to test the inhibitory effect of heparin derivatives on post-surgical metastasis using orthotopically implanted pancreatic cancer in a mouse model. We chose to work with a pancreatic cancer cell line due to its aggressive nature and, by trying to inhibit the surgically induced metastasis in an animal model, we were aiming to develop a study model for increasing the survival rate in human cancer patients after surgical treatment.

\section{Materials and methods}

Tumor cells and test compounds. Luciferase-labeled pancreatic cancer cell line Mpanc96-luc was provided by Dr Thiruvengadam Arumugam (M.D. Anderson Cancer Center, Houston, TX, USA). Tinzaparin, an LMWH, was obtained from Leo Pharma Inc. (Ballerup, Denmark). S-NACH was synthesized at Rensselaer Polytechnic Institute (Rensselaer, NY, USA). Both tinzaparin and S-NACH were solubilized in PBS at concentrations of $10-20 \mathrm{mg} / \mathrm{ml}$. E-cadherin and secondary antibodies were purchased from Santa Cruz Biotechnology Inc. (Dallas, TX, USA).

Animals. Immune-deficient female $\mathrm{NCr}$ nude homozygous mice, aged 5-6 weeks and weighing between 18 and $20 \mathrm{~g}$, were purchased from Harlan Laboratories (Indianapolis, IN, USA). Experiments were performed in compliance with Public Health Service Policy on Humane Care and Use of
Laboratory Animals and approved by the Albany VA Medical Center (Albany, NY, USA) IACUC. All animal studies were conducted in the Albany VA Animal Facility, and mice were maintained under specific pathogen-free conditions, with controlled conditions of temperature $\left(20-24^{\circ} \mathrm{C}\right)$ and humidity (60-70\%) and a 12-h light/dark cycle with ad libitum access to water and food.

Liver metastasis after splenic implantation of tumor cells. Mice were randomly distributed into a control group and 2 treatment groups, with up to 8 mice per group. They were anesthetized with inhaled isoflurane and received subcutaneous (s.c.) injection of PBS (the control) or test compounds (tinzaparin or S-NACH) according to the group. Thirty minutes later, a left lateral abdominal incision was made, and one million Mpanc96-luc cells (suspended in $30 \mu 1$ DMEM media) were injected into the spleen. Animals in the 2 treatment groups received daily s.c. injections (10 or $20 \mathrm{mg} / \mathrm{kg}$ ) of the test compounds and were euthanized after 4 weeks in the first trial and after 2 weeks in a second trial. IVIS images were taken once per week to evaluate the extent of metastasis and to assess the best timing of termination of the experiment, and after termination.

Liver metastasis after excision of pancreatic tumor. Mice were randomly distributed into a control group and 2 treatment groups, with up to 8 mice per group. Mice were anesthetized with inhaled isoflurane, and a half million Mpanc96-luc cancer cells (suspended in $30 \mu \mathrm{l}$ DMEM media) were injected into the pancreatic tail through an abdominal incision. One week later, the pancreatic tumor was surgically removed. Animals received PBS (control) or the test compounds (tinzaparin, S-NACH) 30 min before the tumor excision surgery and daily after that for 3 weeks until they were euthanized. In the first trial animals received $10 \mathrm{mg} / \mathrm{kg}$ of either tinzaparin or S-NACH, but S-NACH concentration was increased to $20 \mathrm{mg} / \mathrm{kg}$ in the second trial. The spread of cancer cells was monitored using IVIS imaging once per week and after termination.

Quantitation of metastasis using IVIS imaging. The IVIS imaging system (Caliper Life Sciences, Hopkinton, MA, USA) is an in vivo imaging technology that was used to measure tumor metastasis (19). The system operates by capturing light emitted from a luminescent source, such as luciferin, in this case Mpanc96-luc. Light is measured by a highly sensitive camera and software. Photographic and luminescence images were taken at constant exposure time. Xenogen IVIS Living Image software (Caliper Life Sciences, version 3.2) was used to quantify non-saturated bioluminescence in regions of interest (ROI). Bioluminescence was quantified as photons/second for each ROI (15).

Bleeding time. Bleeding time in mice was tested as described by Dejana et al (20) and in our previous work [Alshaiban et al (21)] for the above two groups of mice during the period of receiving test compounds. After being on treatment for 1 week, and $24 \mathrm{~h}$ after the last treatment, the mice were anesthetized with isoflurane inhalation. Using a scalpel, $0.5 \mathrm{~cm}$ of the distal end of the tail was transected. The remaining length of tail was immersed immediately into a $37^{\circ} \mathrm{C}$ solution of saline. 

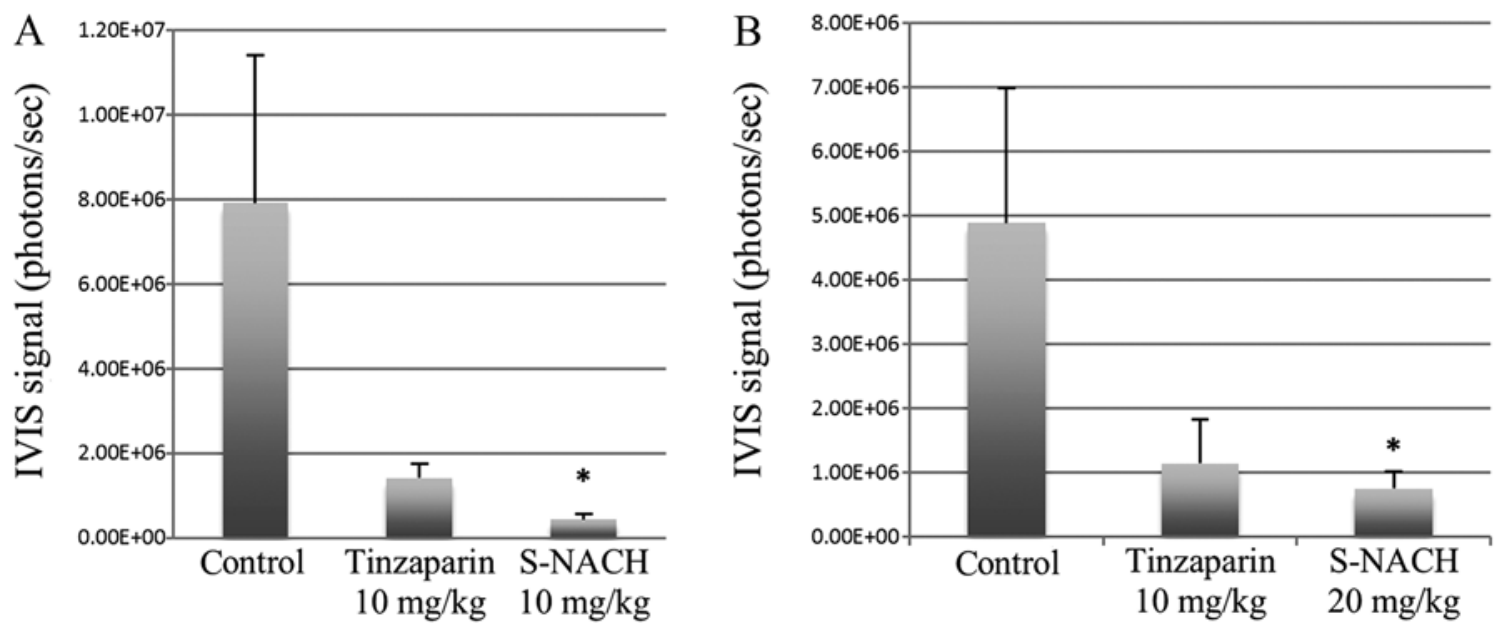

Figure 1. Effect of tinzaparin or S-NACH treatment on tumor metastasis to the liver after splenic implantation of tumor cells. (A) Dose of 10 mg/kg of tinzaparin or S-NACH (number of animals per group =7-8). Mice were sacrificed after 4 weeks, and IVIS imaging was used to quantify metastasis to the liver. There was a significant decrease in bioluminescence emitted by cancer cells for S-NACH relative to the control group (p<0.05). Tinzaparin relative to control was not significant ( $\mathrm{p}=0.10$ ). (B) Dose of $10 \mathrm{mg} / \mathrm{kg}$ of tinzaparin and $20 \mathrm{mg} / \mathrm{kg}$ of S-NACH (number of animals per group = 5-8). Mice were sacrificed after 2 weeks, and IVIS imaging was used to quantify metastasis to the liver. There was a significant decrease in the number of cancer cells relative to the control group for $\mathrm{S}-\mathrm{NACH}(\mathrm{p}=0.02)$. Tinzaparin relative to control was not significant $(\mathrm{p}=0.08)$. Data represent mean $\pm \mathrm{SEM}$. ${ }^{*} \mathrm{p}<0.05$.

Bleeding time was measured from the time of tail transection until visible bleeding could no longer be observed.

Histopathology. Tumor specimens were fixed in $10 \%$ buffered formalin, processed, and embedded in paraffin. After fixation the specimens were transferred into the embedding chambers to hold the specimens in position until the paraffin became solid to prevent further rotation. Four-micrometer serial sections were cut and then stained using haematoxylin and eosin. Sections were evaluated for various pathologic parameters using a light microscope (Leica, Buffalo Grove, IL, USA).

Western blot analysis. MPanc96-luc cells were incubated with S-NACH $(20 \mu \mathrm{g} / \mathrm{ml})$ and cultured for $48 \mathrm{~h}$. Proteins were collected, and concentrations were determined by the Bradford assay using bovine serum albumin as a standard (Protein Assay kit, Bio-Rad Laboratories, Hercules, CA, USA). Total protein extracts $(50 \mu \mathrm{g})$ were mixed with SDS sample buffer $(6.25 \mathrm{mM}$ Tris-HCl, pH 6.8, 2.3\% SDS, $10 \%$ glycerol, $5 \% \beta$-mercaptoethanol, $0.005 \%$ bromophenol blue) and resolved by SDS-PAGE on $10-20 \%$ gradient acrylamide gels. Proteins $(50 \mu \mathrm{g})$ were detected immunologically following semi-dry electro-transfer (Trans-Blot SD system, Bio-Rad Laboratories) onto PVDF membranes (Millipore, Billerica, MA, USA). The membranes were blocked with $5 \%$ non-fat dry milk in Tris-buffered saline with Tween-100 for $30 \mathrm{~min}$ at room temperature and incubated overnight at $4^{\circ} \mathrm{C}$ with antiE-cadherin. After washing 3 times in $0.5 \%$ non-fat dry milk in Tris-buffered saline with Tween-100, blots were incubated with horseradish peroxidase-conjugated secondary antibody for $1 \mathrm{~h}$ at room temperature. Band intensities were measured using Image J software.

Statistical analysis. An overall comparison of the means for all groups (control, tinzaparin, and $\mathrm{S}-\mathrm{NACH}$ ) was carried out using a one-way ANOVA. Tukey confidence intervals were used to test for differences in means for each experimental group (tinzaparin and $\mathrm{S}-\mathrm{NACH}$ ) versus the control group. A value of $\mathrm{p}<0.05$ indicated a statistically significant difference.

\section{Results}

Liver metastasis after splenic implantation of tumor cells. In the first trial of the experiment we compared tinzaparin $(10 \mathrm{mg} / \mathrm{kg})$ and S-NACH $(10 \mathrm{mg} / \mathrm{kg})$ to the control (Fig. 1A). We were able to significantly inhibit the tumor metastasis of Mpanc96-luc cancer cells from spleen to liver for S-NACH relative to the control group $(\mathrm{p}<0.05)$. For tinzaparin relative to control, a trend for metastasis inhibition was evident but not statistically significant $(p=0.1)$. The IVIS images after animal sacrifice are shown in Fig. 2, and both tinzaparin and S-NACH groups had less light intensity of metastasized cancer cells compared to the control group. In the second trial we increased the dose of the well-tolerated S-NACH to $20 \mathrm{mg} / \mathrm{kg}$ s.c. daily. We were again able to show a statistically significant inhibition of tumor metastasis to the liver for S-NACH relative to the control group ( $\mathrm{p}=0.02$, Fig. 1B). For tinzaparin relative to control, a strong trend for metastasis inhibition was evident but did not approach statistical significance $(p=0.08)$. There were no animal deaths in the S-NACH group, but there were 4 deaths $(\sim 50 \%)$ in the tinzaparin group, most probably because of internal bleeding after the surgery.

Liver metastasis after excision of pancreatic tumor. Treatment with $10 \mathrm{mg} / \mathrm{kg}$ of tinzaparin or S-NACH resulted in decreased metastasis to the liver after pancreatic tumor excision, but it was not statistically significant compared to control $(\mathrm{p}=0.90$ for tinzaparin, $\mathrm{p}=0.19$ for S-NACH). However, $10 \mathrm{mg} / \mathrm{kg}$ of $\mathrm{S}-\mathrm{NACH}$ was able to significantly decrease metastasis to the kidneys compared to control ( $\mathrm{p}=0.005$, data not shown) and to 


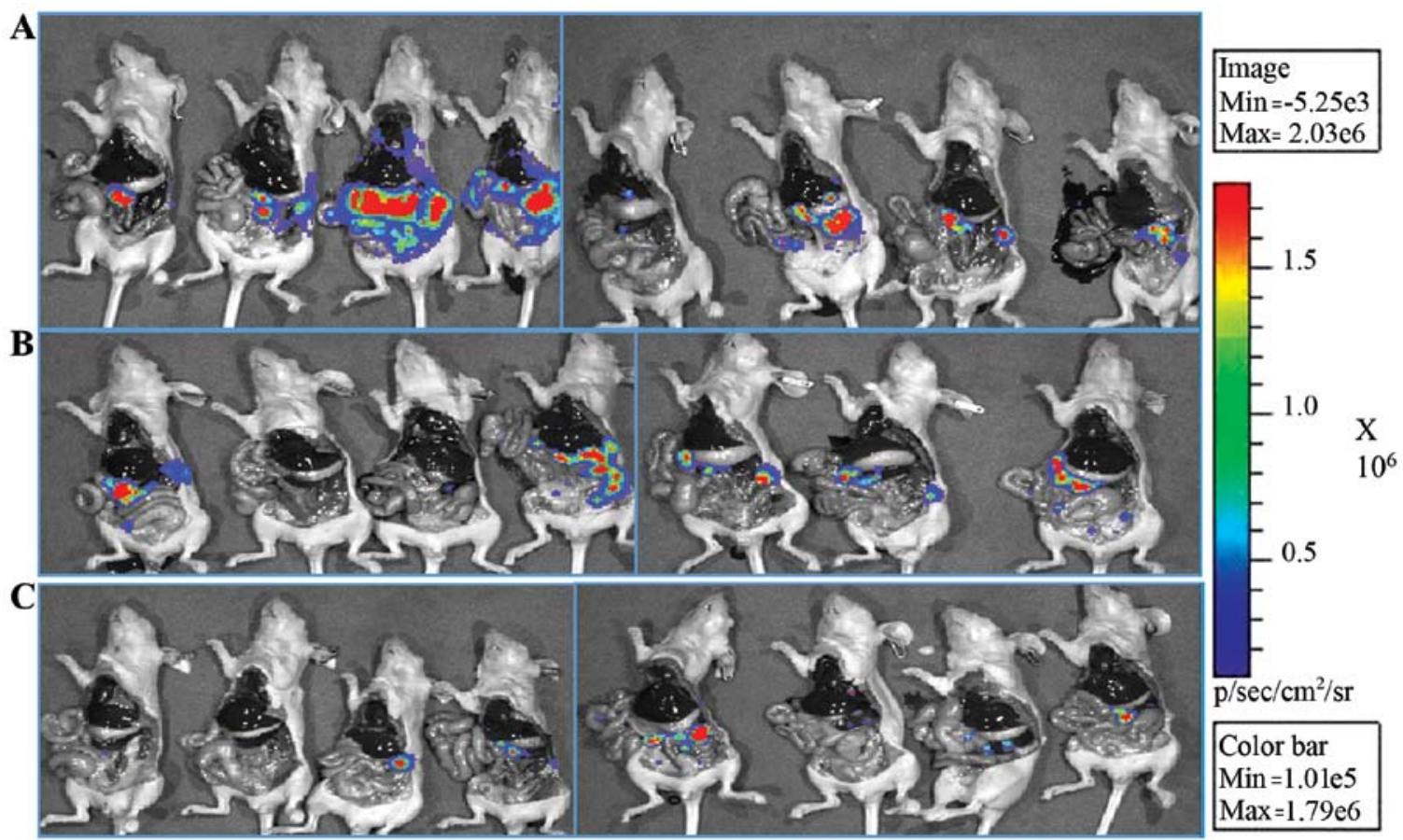

Figure 2. Representative IVIS images demonstrating the effect of tinzaparin or S-NACH treatment on tumor metastasis versus control. Mice were sacrificed after 4 weeks, and these IVIS images taken. (A) Control group received PBS. (B) Tinzaparin (10 mg/kg) group. (C) S-NACH (10 mg/kg) group. It can be seen by visual comparison that the extent of tumor metastasis was decreased by treatment with either tinzaparin or S-NACH.
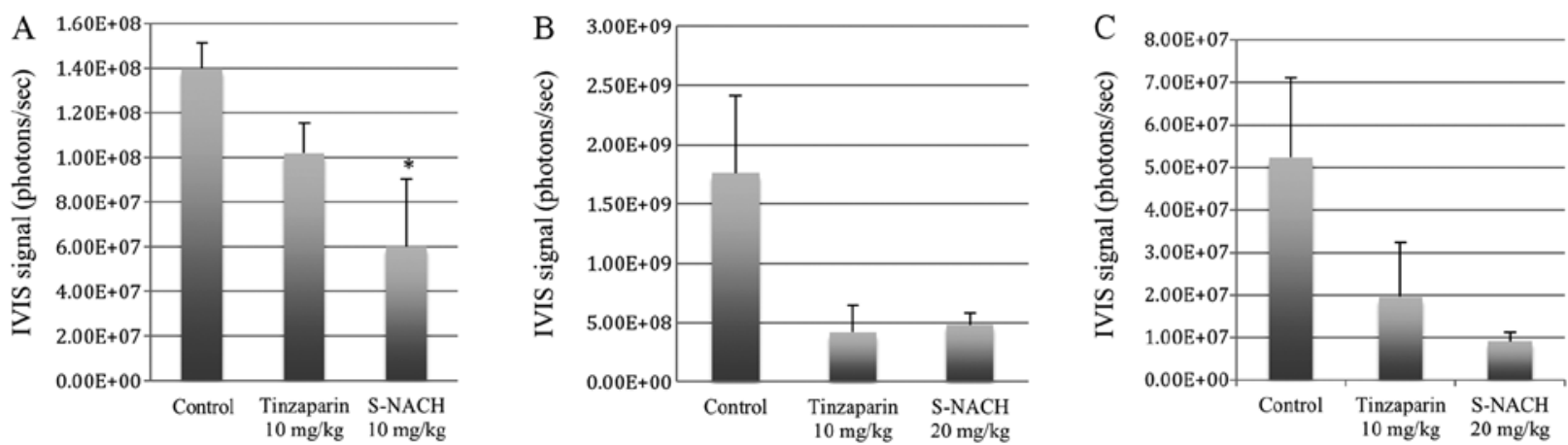

Figure 3. Effect of tinzaparin or S-NACH treatment on tumor recurrence and metastasis after surgical excision of pancreatic tumor. Mice were sacrificed after 3 weeks, and IVIS imaging was used to quantify tumor recurrence or metastasis. (A) A dose of $10 \mathrm{mg} / \mathrm{kg}$ S-NACH inhibited tumor recurrence and showed a significant decrease in the number of cancer cells relative to the control $(\mathrm{p}<0.05), \mathrm{n}=8,4$, and 6 for control, tinzaparin, and S-NACH, respectively. (B) A dose of $20 \mathrm{mg} / \mathrm{kg} \mathrm{S-NACH}$ inhibited tumor recurrence and showed a decrease in the number of cancer cells relative to control, but did not reach statistical significance $(\mathrm{p}=0.1), \mathrm{n}=6,4$, and 6 for control, tinzaparin and S-NACH, respectively. (C) A dose of $20 \mathrm{mg} / \mathrm{kg}$ S-NACH showed a decrease in number of cancer cells metastasizing to the liver, but did not reach statistical significance $(\mathrm{p}=0.08), \mathrm{n}=6,4$, and 6 for control, tinzaparin, and S-NACH, respectively. Data represent mean \pm SEM. ${ }^{*} \mathrm{p}<0.05$.
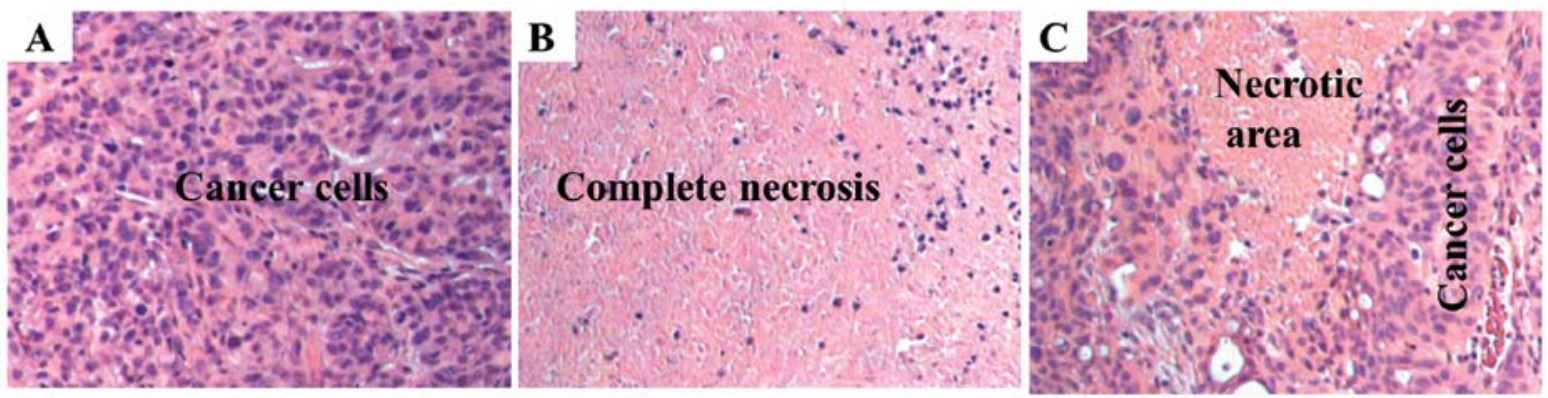

Figure 4. Effect of tinzaparin or S-NACH on MPanc96 tumor necrosis. Representative micrographs of H and E stained histological sections of orthotopic pancreatic tumors. (A) Control group. (B) Treatment with S-NACH $(20 \mathrm{mg} / \mathrm{kg})$. (C) Treatment with tinzaparin (10 mg/kg). Histopathological analysis of the orthotopic pancreatic tumors of MPanc96 cells treated with S-NACH or tinzaparin showed significant increase in necrotic areas versus control $(54 \pm 3 \%$ $\mathrm{S}-\mathrm{NACH}, \mathrm{p}<0.01 ; 31 \pm 4 \%$ tinzaparin, $\mathrm{p}<0.05 ; 16 \pm 5 \%$ control). 

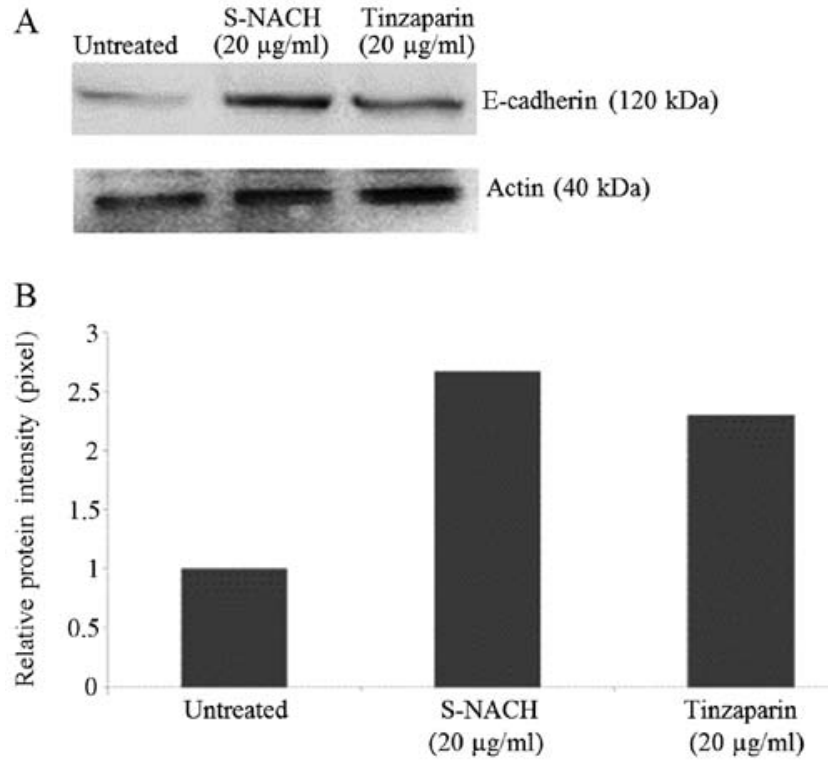

Figure 5. S-NACH increased the expression of E-cadherin protein (A) Western blot analysis showed increased expression of E-cadherin by S-NACH or tinzaparin compared to untreated cells (control). (B) Protein intensity of the bands indicated a 2.0 - to 2.5 -fold increase after treatment with tinzaparin or S-NACH, respectively.

decrease the recurrence of local tumor after surgery compared to control ( $\mathrm{p}<0.05$, Fig. 3A). Treatment with $20 \mathrm{mg} / \mathrm{kg}$ of $\mathrm{S}-\mathrm{NACH}$ resulted in a decrease in tumor recurrence relative to control ( $p=0.1$ Fig. 3B) and a decrease in liver metastasis relative to control ( $\mathrm{p}=0.08$, Fig. $3 \mathrm{C})$. Although these latter results were not statistically significant, the increase in S-NACH concentration from 10 to $20 \mathrm{mg} / \mathrm{kg}$ led to a greater percentage decrease in metastasis (66 vs. $82 \%$, respectively). The percentage of death in the tinzaparin group reached $50 \%$ but did not exceed $11 \%$ in the S-NACH group.

Bleeding time. Tinzaparin treatment of $10 \mathrm{mg} / \mathrm{kg}$ doubled the bleeding time $(124 \pm 21 \mathrm{sec})$ compared to the control group $(60 \pm 18 \mathrm{sec}, \mathrm{p}<0.05)$, and S-NACH treatment of $10 \mathrm{mg} / \mathrm{kg}$ had no effect on bleeding time compared to control. When the dose of S-NACH was increased to $20 \mathrm{mg} / \mathrm{kg}$, there was still no difference in mean bleeding time $(64 \pm 19 \mathrm{sec}, \mathrm{p}=0.74)$.

Histopathology. Histology showed that untreated animals have high-grade (anaplastic) features as common to advanced stage pancreatic cancer (Fig. 4A). In S-NACH treated animals, tumors showed large regions $(50 \%)$ of necrosis $(\mathrm{p}<0.01$, Fig. 4B) when compared to control group (15\%). In contrast, tinzaparin treatment resulted in modest increase in necrotic area (30\%) (Fig. 4C) as compared to control group. Necrotic areas included showed both early stage (fragmented and small nucleus) and late stage (ghost cells without nucleus) areas indicating that S-NACH had effects on early and later aspects of cell death. Tumor necrosis induced by S-NACH was inversely proportional to the bioluminescent signal in the tumor, since only live cells show bioluminescent signal.

Western blot analysis. When MPanc96-luc cells were incubated with either S-NACH or tinzaparin, the expression of
E-cadherin was increased by 2.0 - to 2.5 -fold as compared to control untreated cells (Fig. 5).

\section{Discussion}

The strong involvement of platelets with cancer metastasis was proven a long time ago in studies that showed that thrombocytopenia was consistently associated with a decreased incidence of distant metastases (22). More recently, P-selectin was found to be the link between platelets and cancer cells. P-selectin deficient mice were injected intravenously with cancer cells, lung metastasis decreased, and then injecting those mice with heparin did not show a synergistic effect, thus indicating that heparin and P-selectin work under the same mechanism $(4,23)$.

A meta-analysis in 2007 showed that LMWH decreased mortality in cancer patients $\leq 13.3 \%$ compared to warfarin, which non-significantly reduced mortality to $5.8 \%$ (12). It was shown that the effect of S-NACH in inhibiting metastasis is not related to the heparin anticoagulant property as reported from our laboratory, and by others $(14,15,24)$. These results indicate that the beneficial use of LMWH and its derivatives is not due to its anticoagulant activity. In contrast, the anticoagulant property is a drawback in using heparin, and as shown in our animal model, heparin caused higher mortality in mice due to internal bleeding compared to other groups.

The development of NACH provided a heparin derivative but without the side effect of increased bleeding. Different types of NACH have been prepared and tested; they vary in their efficacy. Kragh et al was able to demonstrate that $\mathrm{NACH}$ inhibited spontaneous metastasis by $48 \%$ compared to $12 \%$ for LMWH (25). In our laboratory, S-NACH demonstrated potent inhibition of pancreatic cancer adhesion, invasion, and metastasis (experimental metastasis) in addition to its inhibitory effects on tumor growth and tumor angiogenesis, which is not the case with other non-anticoagulant heparins (24). In our present experiments we were able to prove again the efficacy of S-NACH in inhibiting experimental metastasis; there was a $72-82 \%$ decrease in liver metastasis compared to control group. S-NACH was also safe and did not increase the death rate among the mice even after increasing the dose.

Although heparin and its derivatives work best on inhibiting hematogenous spread of tumor cells via P-selectinmediated platelets - cancer cell adhesion, heparin possesses other biological activities such as inhibition of angiogenesis and lymphogenesis. Lymphogenesis is one of the mechanisms enhanced by cancer cells to facilitate metastasis. Lymphatic vessels provide a route to local lymph nodes, after which metastases often travel through the blood (26). The increased production of vascular endothelial growth factor (VEGF) stimulates the growth of new blood and lymph vessels. The gastrointestinal carcinomas are known to metastasize first to lymph nodes (27), and inhibiting angiogenesis and lymphogenesis is another biological activity of heparin that aids in preventing metastasis (28).

In vitro studies demonstrated that tumor cells could be shed during surgical manipulation of the primary tumor (29). Retrospective clinical studies showed more favorable results for patients receiving perioperative LMWH (17). Von Tempelhoff et al showed the clinically beneficial effect of preoperative heparin treatment (18). Here, treatment with 
LMWH or S-NACH before performing the surgery on the mice allowed the test compounds time to bind to the P-selectin platelets and inhibit them from binding to the disseminated cancer cells. The results we obtained using S-NACH showed a decrease in cancer recurrence at the site of surgical removal compared to control $(\mathrm{p}<0.05)$, and although it showed a trend in decreasing liver metastasis, the number did not reach statistical significance $(\mathrm{p}=0.06)$. This was probably due to the small number of mice that survived after undergoing two surgeries in a week (implanting the cancer cells and excising the tumor). S-NACH demonstrated distinct upregulation of key junctional adhesion molecule E-cadherin; its upregulation is known to limit cancer cell migration and invasion (30).

The benefits of heparin derivative in inhibiting metastasis can be introduced into clinical practice and can be used synergistically with current chemotherapies. LMWH and S-NACH were shown to increase the uptake of chemotherapeutics in treating breast cancer in a mouse model (31). This effect was also shown in a retrospective study of combining heparin and chemotherapy for optimal results in treating lung cancer patients (32). Research has shown that a brief course of subcutaneous low molecular weight heparin favorably influences the survival in patients with advanced malignancy, and it deserves additional clinical evaluation (33). Lebeau et al carried out a randomized, multicenter clinical trial in 1994 to study the positive influence of anticoagulant treatment in small cell lung cancer. The study included 277 patients, and the results showed better survival rates for patients treated with subcutaneous heparin for 5 weeks compared to control group at 1,2, and 3 years (40 vs. 30,11 vs. 9 and 9 vs. $6 \%$, respectively) (34).

The average dose of the LMWH dalteparin as an antimetastatic agent in human clinical trials was 5,000 IU, equivalent to $3-5 \mathrm{mg} / \mathrm{kg}$ in humans (12), and the average dose of enoxaparin in humans is $1.0-1.5 \mathrm{mg} / \mathrm{kg}$. In our experiment we used $10 \mathrm{mg} / \mathrm{kg}$ for LMWH (tinzaparin) and as high as $20 \mathrm{mg} / \mathrm{kg}$ for S-NACH. S-NACH was safely administered and did not increase the bleeding time compared to control group. The number of mouse deaths in all experiments was calculated at the end of the project, and we noted a high percentage of mice dying in the LMWH group due to internal bleeding, whereas the percentage of death in the S-NACH group was statistically similar to the control. Further studies might be required to firm these trends with a larger sample size and perhaps reducing the dose of tinzaparin to $5 \mathrm{mg} / \mathrm{kg}$.

Potential mechanisms for the anticancer efficacy of heparin derivatives, including S-NACH or tinzaparin, might be due to their multimodal mechanisms contributing to anticancer and anti-metastasis efficacy. This might include their effective antiangiogenesis efficacy (via the release of endogenous endothelial TFPI), inhibition of cancer cell adhesion (anti-selectin), inhibition of cancer cell invasion (inhibition of heparinases, matrix degrading enzymes, and through other cell adhesion molecules), anti-inflammatory efficacy, and possibly other mechanisms (35-38). It was also suggested that combining the effect of heparin and its derivative S-NACH with current adjuvant or neo-adjuvant therapy will lead to a decrease in the required chemotherapy dose and increased tumor chemo-responsiveness based on reported studies from our laboratories (31).

These data suggest that S-NACH is an effective anti-metastatic agent in our mouse model; it decreased distal metastasis and surgically induced metastasis. S-NACH was also found to be safe in terms of bleeding tendencies compared to LMWH. $\mathrm{S}-\mathrm{NACH}$ warrants further clinical evaluation.

\section{Acknowledgements}

We appreciate the excellent editing by Dr Kelly Keating [Pharmaceutical Research Institute (PRI) at the Albany College of Pharmacy and Health Sciences] and the technical support by staff of the PRI. We thank Alexander Durant in the Animal Laboratory of the Albany Stratton VA Medical Center, (Albany, NY, USA); Dr Thiruvengadam Arumugam (M.D. Anderson Cancer Center, Houston, TX, USA) for supplying the Mpanc96-luc cell line; and Dr Robert J. Linhardt (Rensselaer Polytechnic Institute, Rensselaer, NY, USA) for supplying S-NACH. S.A. Mousa holds a US patent on S-NACH (13).

\section{References}

1. Siegel R, Ward E, Brawley O and Jemal A: Cancer statistics, 2011: the impact of eliminating socioeconomic and racial disparities on premature cancer deaths. CA Cancer J Clin 61: 212-236, 2011.

2. Chambers AF, Groom AC and MacDonald IC: Dissemination and growth of cancer cells in metastatic sites. Nat Rev Cancer 2: 563-572, 2002.

3. Sener SF, Fremgen A, Menck HR and Winchester DP: Pancreatic cancer: a report of treatment and survival trends for 100,313 patients diagnosed from 1985-1995, using the National Cancer Database. J Am Coll Surg 189: 1-7, 1999.

4. Borsig L, Wong R, Feramisco J, Nadeau DR, Varki NM and Varki A: Heparin and cancer revisited: mechanistic connections involving platelets, P-selectin, carcinoma mucins, and tumor metastasis. Proc Natl Acad Sci USA 98: 3352-3357, 2001.

5. Stone JP and Wagner DD: P-selectin mediates adhesion of platelets to neuroblastoma and small cell lung cancer. J Clin Invest 92: 804-813, 1993.

6. Mannori G, Crottet P, Cecconi O, et al: Differential colon cancer cell adhesion to E-, P-, and L-selectin: role of mucin-type glycoproteins. Cancer Res 55: 4425-4431, 1995.

7. Nelson RM, Cecconi O, Roberts WG, Aruffo A, Linhardt RJ and Bevilacqua MP: Heparin oligosaccharides bind L- and P-selectin and inhibit acute inflammation. Blood 82: 3253-3258, 1993.

8. Koenig A, Norgard-Sumnicht K, Linhardt R and Varki A: Differential interactions of heparin and heparan sulfate glycosaminoglycans with the selectins. Implications for the use of unfractionated and low molecular weight heparins as therapeutic agents. J Clin Invest 101: 877-889, 1998.

9. Zacharski LR and Loynes JT: The heparins and cancer. Curr Opin Pulm Med 8: 379-382, 2002.

10. Kasthuri RS, Taubman MB and Mackman N: Role of tissue factor in cancer. J Clin Oncol 27: 4834-4838, 2009.

11. Capila I and Linhardt RJ: Heparin-protein interactions. Angew Chem Int Ed Engl 41: 391-412, 2002.

12. Kuderer NM, Khorana AA, Lyman GH and Francis CW: A meta-analysis and systematic review of the efficacy and safety of anticoagulants as cancer treatment: impact on survival and bleeding complications. Cancer 110: 1149-1161, 2007.

13. Mousa SA: Oxidized heparin fractions and their use in inhibiting angiogenesis. US patent 8,071,569. 2011. December 06, 2011.

14. Lapierre F, Holme K, Lam L, et al: Chemical modifications of heparin that diminish its anticoagulant but preserve its heparanase-inhibitory, angiostatic, anti-tumor and anti-metastatic properties. Glycobiology 6: 355-366, 1996.

15. Sudha T, Phillips P, Kanaan C, Linhardt RJ, Borsig L and Mousa SA: Inhibitory effect of non-anticoagulant heparin (S-NACH) on pancreatic cancer cell adhesion and metastasis in human umbilical cord vessel segment and in mouse model. Clin Exp Metastasis 29: 431-439, 2012.

16. van der Bij GJ, Oosterling SJ, Beelen RH, Meijer S, Coffey JC and van Egmond $\mathrm{M}$ : The perioperative period is an underutilized window of therapeutic opportunity in patients with colorectal cancer. Ann Surg 249: 727-734, 2009. 
17. Kakkar A, Hedges R, Williamson R and Kakkar V: Perioperative heparin-therapy inhibits late death from metastatic cancer. Int J Oncol 6: 885-888, 1995 .

18. von Tempelhoff GF, Harenberg J, Niemann F, Hommel G, Kirkpatrick CJ and Heilmann L: Effect of low molecular weight heparin (Certoparin) versus unfractionated heparin on cancer survival following breast and pelvic cancer surgery: a prospective randomized double-blind trial. Int J Oncol 16: 815-824, 2000.

19. Lim E, Modi KD and Kim J: In vivo bioluminescent imaging of mammary tumors using IVIS spectrum. J Vis Exp 26: 1210, 2009

20. Dejana E, Callioni A, Quintana A and de Gaetano G: Bleeding time in laboratory animals. II - A comparison of different assay conditions in rats. Thromb Res 15: 191-197, 1979.

21. Alshaiban A, Muralidharan-Chari V, Nepo A and Mousa SA Modulation of sickle red blood cell adhesion and its associated changes in biomarkers by sulfated non-anticoagulant heparin derivative. Clin Appl Thromb Hemost (In press).

22. Gasic GJ, Gasic TB and Stewart CC: Antimetastatic effects associated with platelet reduction. Proc Natl Acad Sci USA 61: 46-52, 1968.

23. Kim YJ, Borsig L, Varki NM and Varki A: P-selectin deficiency attenuates tumor growth and metastasis. Proc Natl Acad Sci USA 95: 9325-9330, 1998.

24. Mousa SA, Linhardt R, Francis JL and Amirkhosravi A: Antimetastatic effect of a non-anticoagulant low-molecular-weight heparin versus the standard low-molecular-weight heparin, enoxaparin. Thromb Haemost 96: 816-821, 2006.

25. Kragh M, Binderup L, Vig Hjarnaa PJ, Bramm E, Johansen KB and Frimundt Petersen C: Non-anti-coagulant heparin inhibits metastasis but not primary tumor growth. Oncol Rep 14: 99-104, 2005.

26. Bacac M and Stamenkovic I: Metastatic cancer cell. Annu Rev Pathol 3: 221-247, 2008.

27. Kopfstein L and Christofori G: Metastasis: cell-autonomous mechanisms versus contributions by the tumor microenvironment. Cell Mol Life Sci 63: 449-468, 2006.

28. Achen MG, Mann GB and Stacker SA: Targeting lymphangiogenesis to prevent tumour metastasis. Br J Cancer 94: 1355-1360, 2006 .
29. Ito S, Nakanishi H, Hirai T, et al: Quantitative detection of CEA expressing free tumor cells in the peripheral blood of colorectal cancer patients during surgery with real-time RT-PCR on a LightCycler. Cancer Lett 183: 195-203, 2002.

30. Wang F, Sloss C, Zhang X, Lee SW and Cusack JC: Membranebound heparin-binding epidermal growth factor like growth factor regulates E-cadherin expression in pancreatic carcinoma cells. Cancer Res 67: 8486-8493, 2007.

31. Phillips PG, Yalcin M, Cui H, et al: Increased tumor uptake of chemotherapeutics and improved chemoresponse by novel nonanticoagulant low molecular weight heparin. Anticancer Res 31: 411-419, 2011.

32. Lebeau B, Baud M, Masanes MJ, Febvre M, Mokhtari T and Chouaid C: Optimization of small-cell lung cancer chemotherapy with heparin: a comprehensive retrospective study of 239 patients treated in a single specialized center. Chemotherapy 57: 253-258, 2011.

33. Klerk CP, Smorenburg SM, Otten HM, et al: The effect of low molecular weight heparin on survival in patients with advanced malignancy. J Clin Oncol 23: 2130-2135, 2005

34. Lebeau B, Chastang C, Brechot JM, et al: Subcutaneous heparin treatment increases survival in small cell lung cancer. 'Petites Cellules' Group. Cancer 74: 38-45, 1994.

35. Mousa SA and Petersen LJ: Anti-cancer properties of low-molecular-weight heparin: preclinical evidence. Thromb Haemost 102: 258-267, 2009

36. Mousa SA: Comparative pharmacodynamic assessment of the antiangiogenesis activity of heparin and low-molecular-weight heparin fractions: structure-function relationship. Clin Appl Thromb Hemost 19: 48-54, 2013.

37. Yu CJ, Ye SJ, Feng ZH, et al: Effect of Fraxiparine, a type of low molecular weight heparin, on the invasion and metastasis of lung adenocarcinoma A549 cells. Oncol Lett 1: 755-760, 2010.

38. Ilan N, Elkin M and Vlodavsky I: Regulation, function and clinical significance of heparanase in cancer metastasis and angiogenesis. Int J Biochem Cell Biol 38: 2018-2039, 2006. 